\title{
Effects of the Structure and Composition of Montmorillonite on the Dimerization of Unsaturated Fatty Acids
}

\author{
Xue Huang, Yaoqi Yin, Buning Zhang and Guangzhu Feng* \\ College of Chemistry and Chemical Engineering, Zhongkai University of Agriculture and Engineering, \\ 510225 Guangzhou, P. R. China
}

\begin{abstract}
The effects of structure and composition of different montmorillonites on their catalytic performance in the dimerization of unsaturated fatty acids were investigated. The dimerization reaction was performed at $240{ }^{\circ} \mathrm{C}$ for $3 \mathrm{~h}$, with montmorillonite as catalyst, and $\mathrm{LiCl}$ as the co-catalyst. The results showed that the specific surface areas of the different montmorillonites did not have a major effect on their catalytic performance, but the interlayer spacing and surface acidity of montmorillonite show significant effects on the product yield. After the organic modification through hexadecyl trimethyl ammonium bromide (CTAB) intercalation, the microenvironment of the montmorillonite layer changes. Moreover, the inner and outer surfaces change from being hydrophilic to lipophilic, which improves the dispersibility of montmorillonite in fatty acids. The $\mathrm{x}_{\mathrm{Al}} / \mathrm{x}_{\mathrm{Si}}$ molar ratios of the three samples are similar and lie within the range of 0.4306-0.5010, thus the $\mathrm{x}_{\mathrm{Al}} / \mathrm{x}_{\mathrm{Si}}$ molar ratio has little effect on the dimerization reaction.
\end{abstract}

Keywords: montmorillonite, unsaturated fatty acid, dimerization, dimer acid

\section{Introduction}

Oligomer of unsaturated fatty acid is a major chemical raw material, especially dimer acids and their derivatives, with excellent physical and chemical properties, and widely used for the preparation of polyamide resin, ${ }^{1,2}$ lubricants, ${ }^{3,4}$ adhesives, ${ }^{5}$ corrosion inhibitor, ${ }^{6}$ inks ${ }^{7,8}$ and other products. The polymerization of unsaturated fatty acids is mainly divided into two groups: high temperature thermal polymerization and catalytic polymerization. The latter has the advantages of superior selectivity and relatively moderate reaction conditions, while the former is widely used to produce dimer acids in industry. There are two catalytic processes for the synthesis of dimer acids, which include homogeneous catalysis and heterogeneous catalysis. The traditional catalytic process used for the polymerization of dimer acids is homogeneous catalysis, where the catalyst has alkali or alkaline metal salts, iodine, Lewis and Brønsted acids. Compared to the homogeneous catalysis, heterogeneous catalysis is more environmentally friendly, widely available, inexpensive, easy to separate from the products and reuse, and commercially more attractive. Catalysts used in heterogeneous catalytic processes are mainly clays and clay materials, such as kaolinite,

*e-mail: fengguangzhu@163.com montmorillonite (MMT), sepiolite and bentonite. Based on the mechanism of Diels-Alder reaction ${ }^{9}$ and carbenium ion polymerization, ${ }^{10}$ the suitable catalysts for preparation of dimer acids are zirconia, ${ }^{11}$ molecular sieves, ${ }^{12,13}$ Lewis acids, ${ }^{14}$ ionic liquids and so on. ${ }^{15}$ The dimerization reaction with these catalysts has many side reactions and low polymer conversion rate. Only bentonite clay is currently being applied for industrial preparation of dimer acids.

Montmorillonite (MMT) is the most commonly used clay mineral because of its natural abundance and beneficial properties (high cationic exchange capacity, high specific surface area, and large aspect ratio). ${ }^{16}$ The main elements present on MMT are $\mathrm{Si}$ and $\mathrm{Al}$, and the chemical formula is $\left(\mathrm{Al}_{2-\mathrm{x}} \mathrm{Mg}_{\mathrm{x}}\right) \mathrm{Si}_{4} \mathrm{O}_{10}(\mathrm{OH})_{2} \bullet\left(\mathrm{M} \bullet \mathrm{nH}_{2} \mathrm{O}\right)$ ( where $\mathrm{M}=\mathrm{Na}^{+}, \mathrm{Ca}^{2+}$, $\mathrm{Mg}^{2+}$, etc.); it is a typical 2:1 structure with three sheets (octahedral/tetrahedral/octahedral). MMT surfaces are negatively charged due to the tetrahedral and octahedral substitution of $\mathrm{Mg}^{2+}$ or $\mathrm{Fe}^{2+}$ ions by $\mathrm{Al}^{3+}$ ions. The negative charge of MMT surfaces is balanced by the cations $\left(\mathrm{Li}^{+}\right.$, $\mathrm{Na}^{+}, \mathrm{K}^{+}, \mathrm{Ca}^{2+}, \mathrm{Mg}^{2+}$ ) coordinated between MMT layers surrounded by the water molecules. ${ }^{17}$ Hydrated state of the interlayer cations and $\mathrm{Si}-\mathrm{OH}$ groups at the edges of the MMT minerals make the MMT surfaces hydrophilic. The interlayer cations are exchangeable with each other and they are also exchangeable with organic cations such as primary, secondary and quaternary ammonium 
compounds. ${ }^{18,19}$ Cation exchange capacity (CEC) is an important characteristic of the MMT minerals, which represents the degree to which hydrophilicity of MMT can be converted into hydrophobicity or organophilicity, where the organic cations are exchanged with interlayer cations. ${ }^{20}$

After modification, the interlayer spacing of MMT is enlarged, which can provide suitable reaction space for the dimerization of unsaturated fatty acids and effectively prevent polyreaction. In addition, replacement of Si atoms by $\mathrm{Al}$ atoms in MMT can produce two kinds of acid centers, namely, Brønsted acids and Lewis acids. Lewis acids can catalyze the conversion of unconjugated linoleic acid into conjugated linoleic acid, while Brønsted acids can generate carbocations of conjugated linoleic acid, which contributes to the dimerization of unsaturated fatty acid..$^{21}$ Thus, MMT with both Brønsted and Lewis acid sites has excellent catalytic performance for dimerization reaction.

In MMT catalyzed reactions, generally about $10-20 \%$ (mass fraction) of MMT is used. Due to the limited economic benefits from recycling the MMT catalyst, it is generally sent to waste at the end of the reaction. This results in wastage of raw materials and products, as well as environmental pollution. To solve this problem, it is important to develop an eco-friendly catalyst with higher catalytic activity. As MMT catalyst provides reliable yield and quality products, reducing the amount of MMT catalyst would also be a suitable option. Due to the hydrophobicity of MMT, its dispersibility in fatty acids is low, which is unfavorable for polymerization. In this paper, to improve the catalytic activity and lower the dosage of MMT, one-step modified MMT and two-step modified MMT were prepared by organic modification. The catalytic performance of pristine and modified MMT samples in unsaturated fatty acid dimerization was investigated. The structure and properties of the modified MMT were examined by X-ray diffraction (XRD), scanning electron microscopy (SEM), Fourier transform infrared spectroscopy (FTIR), transmission electron microscopy (TEM), pyridine adsorption Fourier transform infrared spectroscopy (Py-IR), energy-dispersive $\mathrm{X}$-ray spectroscopy (EDS) and thermogravimetric analysis (TGA) methods. The findings of this work provide insights into the effects of MMT composition and structure on the dimerization of unsaturated fatty acids, as well as theoretical basis for the selection of catalysts.

\section{Experimental}

\section{Materials}

Mg-montmorillonite (Mg-MMT) with cation exchange capacity (CEC) of 85 meq per $100 \mathrm{~g}$ was purchased and used as catalyst (Zhejiang Fenghong Clay Chemical Co., Ltd., Zhejiang, China). The single particle size is near $30 \mu \mathrm{m}$, BET (Brunauer-Emmett-Teller) surface area is $12.84 \mathrm{~m}^{2} \mathrm{~g}^{-1}$. $\mathrm{LiCl}(>99 \%)$ was purchased and used as co-catalyst (Energy Chemical, Shanghai, China). Hexadecyl trimethyl ammonium bromide (CTAB, $\geq 99 \%$ ) was purchased and used as modifier (Aladdin Industrial Corporation, Shanghai, China). Unsaturated fatty acid (main fatty acid components: $\mathrm{C}_{16: 0} 1.5 \%, \mathrm{C}_{18: 0} 3.9 \%, \mathrm{C}_{18: 1} 33.1 \%, \mathrm{C}_{18: 2} 59.2 \%, \mathrm{C}_{18: 3} 2.3 \%$ ) was purchased as raw material (Liancheng Baixin Science and Technology Co., Ltd., Fujian, China). Deionized water was used for the experiments where mentioned.

\section{CTAB modification of Mg-MMT}

\section{One-step intercalated MMT}

In a typical preparation of the one-step intercalated MMT, $10 \mathrm{~g}$ Mg-MMT was first added to $300 \mathrm{~mL}$ of deionized water and mixed at $500 \mathrm{rpm}$ in a water bath at $70 \pm 1^{\circ} \mathrm{C}$ for $30 \mathrm{~min}$ in order to obtain high swelling value. $\mathrm{CTAB}$ was selected as the intercalation agent which was added to the Mg-MMT dispersion under vigorous stirring at $70 \pm 1^{\circ} \mathrm{C}$ for $3 \mathrm{~h}$. The final mixture was centrifuged and subsequently washed with deionized water until free from halide ion (tested by $0.01 \mathrm{~mol} \mathrm{~L}^{-1} \mathrm{AgNO}_{3}$ solution, until there was no white precipitate). The as-obtained one-step intercalated MMT (marked as O-MMT) was dried at $80^{\circ} \mathrm{C}$ for $24 \mathrm{~h}$ and pulverized to pass through 50,150 and 300 mesh sieves.

\section{Two-step intercalated MMT}

After the first intercalation treatment, $10 \mathrm{~g}$ O-MMT was added to $300 \mathrm{~mL}$ of deionized water and mixed at $500 \mathrm{rpm}$ in a water bath at $60 \pm 1{ }^{\circ} \mathrm{C}$ for $30 \mathrm{~min}$ in order to obtain high swelling value. Then, CTAB was added to the O-MMT dispersion under vigorous stirring. The mixture was stirred at $60 \pm 1^{\circ} \mathrm{C}$ for $3 \mathrm{~h}$. The final mixture was centrifuged and subsequently washed with deionized water until free from the halide ion. The obtained two-step intercalated MMT (denoted as T-MMT) was dried at $80{ }^{\circ} \mathrm{C}$ for $24 \mathrm{~h}$ and pulverized to pass through 50,150 and 300 mesh sieves.

\section{Dimer acid syntheses}

The dimerization experiments were performed in a $250 \mathrm{~mL}$ three-necked flask. The flask was flushed several times with nitrogen at room temperature before reaction and was heated to the specified reaction temperature at ambient pressure. The catalyst and co-catalyst were injected as a slurry along with the reactants only when the 
reaction temperature was attained. This results in a more well-defined starting point of the reaction. After completion of the reaction, the crude product was filtered to remove MMT. The content of dimer acids in the crude product was analyzed by high-performance liquid chromatography (HPLC), and the yield was calculated.

Apart from the MMT catalyst, the as-prepared O-MMT and T-MMT catalysts were also used in separate experiments to synthesize the dimer acid, for comparison purpose. The yield and selectivity of the dimer acids were calculated using equations 1 and 2 , respectively.

Yield $(\%)=\frac{\text { molar amount of the dimer acid }}{\text { molar amount of unsaturated fatty acid }}$

Selectivity $(\%)=\frac{\text { molar amount of the dimer acid }}{\text { molar amount of reaction of unsaturated fatty acid }}$

\section{Measurements}

The content of dimer acids in the product was analyzed by HPLC (Agilent 1200, USA). The mobile phase was a mixture of methanol and water $(9: 1, \mathrm{v} / \mathrm{v})$ with a flow rate of $1.0 \mathrm{~mL} \mathrm{~min}{ }^{-1}$. The detection wavelength was $211 \mathrm{~nm}$ and column temperature was $30^{\circ} \mathrm{C}$.

XRD patterns were obtained using Empyrean diffractometer (PANalytical, Holland) at a wavelength $\lambda=1.5418 \AA, \mathrm{Cu} \mathrm{K \alpha}$ radiation, $40 \mathrm{kV}$ and $40 \mathrm{~mA}$. Divergence slit size was $1 / 32 \AA$. The scanning speed was $40 \mathrm{~s}$ per insert from $1.2^{\circ}-40^{\circ}$ in insert-length of $0.02^{\circ}$. The crystallographic basal distance (d) was calculated for MMT according to Bragg's equation, $\mathrm{n} \lambda=2 \mathrm{~d} \sin \theta$ ( $\theta$ is the diffraction angle, $\mathrm{n}=1, \lambda=1.5418 \AA$ ).

FTIR spectra were recorded with a Spectrum 2000 spectrophotometer (PerkinElmer, USA) to study the curving change of MMT and O-MMT. The powder samples were analyzed in $\mathrm{KBr}$ and scanned from 400 to $4000 \mathrm{~cm}^{-1}$.

SEM was performed using KYKY-2800B (Inspect FEI, Netherlands) to study the morphology of MMT. The powder samples were given a gold coating.

TEM was carried out on a JEM-2010HR Diffractometer (JEM, Japan) at $200 \mathrm{kV}$ to analyze the microstructures of Na-MMT, first-step modified MMT and second-step modified MMT.

The surface areas were determined by $\mathrm{N}_{2}$ adsorption at $77 \mathrm{~K}$ using ASAP 2020 instrument (Micromeritics, USA). The samples were outgassed in vacuum for $12 \mathrm{~h}$ at $110^{\circ} \mathrm{C}$ prior to nitrogen adsorption. The specific surface area was calculated using the BET method based on adsorption data in the partial pressure $\left(\mathrm{P} / \mathrm{P}_{0}\right)$ range of $0-0.1$ and the pore diameter and pore volume were determined from desorption isotherms using the Barrett-Joyner-Halenda (BJH) method.

The thermal stability of MMT was determined on a thermogravimetric analyzer (TG209, NETZSCH, Germany) heated from 0 to $700{ }^{\circ} \mathrm{C}$ under nitrogen atmosphere $\left(20 \mathrm{~mL} \mathrm{~min}^{-1}\right)$ at a heating rate of $20^{\circ} \mathrm{C} \mathrm{min}{ }^{-1}$.

Surface acid sites of MMT were studied by in situ pyridine adsorption infrared spectroscopy. The samples were made into homogeneous tablets and placed in the in situ pool. After degassing for $2 \mathrm{~h}$ at $120^{\circ} \mathrm{C}$ and $2-10 \mathrm{~Pa}$, the excess pyridine probe molecules were injected into the in situ pool after cooling to room temperature. After adsorption equilibrium at a temperature not higher than room temperature, the sample was vacuum degassed for $1 \mathrm{~h}$. The measured wavelength range was $1000-1800 \mathrm{~cm}^{-1}$.

The molar amounts of $\mathrm{Al}$ and $\mathrm{Si}$ elements on the surface of MMT were measured by EDS (Oxford Instruments 51-XMX0003, United Kingdom). The samples were sprayed with gold at the working voltage of $20 \mathrm{kV}$ and the current of $15 \mathrm{~mA}$.

For the dispersion test, $20 \mathrm{~mL}$ of dispersing medium was added into a $60 \mathrm{~mL}$ stoppered flask. Then, $0.4 \mathrm{~g}$ of MMT was weighed into the flask (the concentration of MMT was $2.0 \mathrm{wt} . \%$ ) and magnetic stirring was carried out for $15 \mathrm{~min}$ at room temperature. The dispersion was transferred to a $10 \mathrm{~mL}$ colorimetric tube $(13 \mathrm{~cm}$ in length and $1.5 \mathrm{~cm}$ in diameter), and the stability of the dispersion was observed and recorded.

\section{Results and Discussion}

\section{$\mathrm{XRD}$ analysis}

The differences between pristine MMT, O-MMT and T-MMT can be observed from the XRD patterns (Figure 1). After replacing the intercalated hydrated cations by CTAB cations, the 001 diffraction peaks shifted to the left. The XRD pattern of Mg-MMT shows only one major broad peak, while both O-MMT and T-MMT show two peaks below $6^{\circ}$, with the high intensity peaks at $2 \theta=2-3^{\circ}$. The basal distances of the O-MMT and T-MMT samples significantly expanded after modification, which clearly indicates that the intercalation agent was successfully inserted into the interlayer space of Mg-MMT. However, compared to O-MMT, there was less obvious shift of the 001 diffraction peaks of the T-MMT to the left. The 001 diffraction peaks for Mg-MMT, O-MMT and T-MMT are located at $2 \theta=5.97^{\circ}, 2.33^{\circ}$ and $2.31^{\circ}$, respectively, corresponding to basal distance. The results of yield and selectivity of the dimer acid for the three MMT samples are shown in Table 1. 
Table 1. The basal distances and catalytic properties of MMT

\begin{tabular}{|c|c|c|c|c|}
\hline MMT & $2 \theta /$ degree & Basal distance / nm & Selectivity of dimer acids / \% & Yield of dimer acids / \% \\
\hline Mg-MMT & 5.97 & 1.48 & 73.10 & 32.69 \\
\hline O-MMT & 2.33 & 3.80 & 72.19 & 50.86 \\
\hline T-MMT & 2.31 & 3.82 & 72.77 & 68.03 \\
\hline
\end{tabular}

O-MMT: one-step intercalated MMT; T-MMT: two-step intercalated MMT.

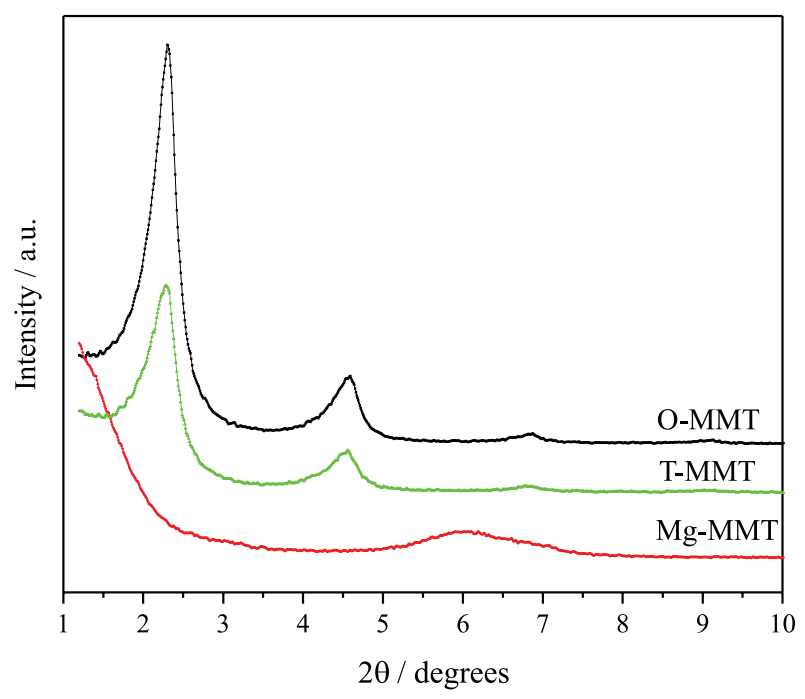

Figure 1. XRD patterns of Mg-MMT, O-MMT and T-MMT.

The results of Table 1 show that the synthesis of dimer acid catalyzed by modified MMT samples is better than that catalyzed by the unmodified MMT. The basal distances of O-MMT and T-MMT are the same, but the catalytic performance of T-MMT is better than that of O-MMT. The small basal distance of the unmodified MMT and strong hydrophilicity were likely not conducive to the entry and adsorption of unsaturated fatty acid molecules. On the other hand, the basal distance and hydrophobicity of O-MMT and T-MMT are enhanced after modification and thus, the unsaturated fatty acid molecules could easily enter the layers of MMT and participate in the reaction. Thus, both the organic modified MMT samples showed good catalytic performance, and especially the two-step intercalated MMT.

\section{FTIR analysis}

The FTIR spectra of the modified MMT catalyst also showed some differences from that of Mg-MMT (Figure 2). The hydroxyl $(-\mathrm{OH})$ stretching vibration of the characteristic structure of Mg-MMT appeared at $3620 \mathrm{~cm}^{-1}$ as a broad band, while the $-\mathrm{OH}$ bending vibration band occurred at $1639 \mathrm{~cm}^{-1}$. Moreover, $\mathrm{Si}-\mathrm{O}$ stretching vibration band was observed at 1101 and $1032 \mathrm{~cm}^{-1}$, and the interlayer water stretching vibration appeared at $3450 \mathrm{~cm}^{-1}$. It was proved by the decrease in the intensity of $3620 \mathrm{~cm}^{-1}$, which is due to the removal of octahedral cations causing the loss of water and hydroxyl groups coordinated to them. Curves of other samples showed the characteristic peaks of Mg-MMT, indicating that the layered structure of MMT was not destroyed after modification.

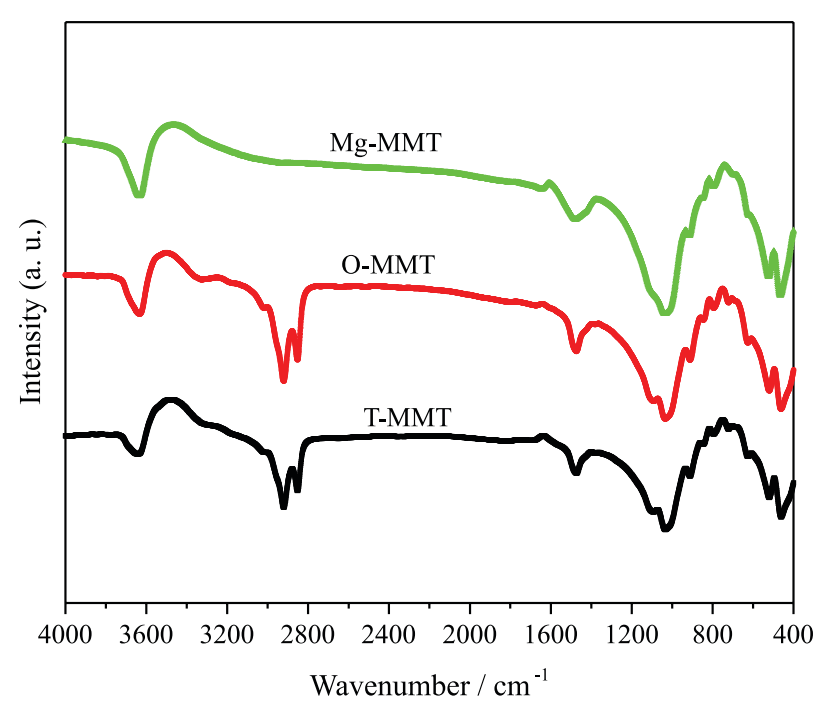

Figure 2. FTIR (KBr) spectra of Mg-MMT, O-MMT and T-MMT.

Compared with the FTIR spectrum of Mg-MMT, several distinct changes were observed in that of O-MMT and T-MMT from 3000 to $2800 \mathrm{~cm}^{-1}$. Absorption peaks were created at 2850 and $2918 \mathrm{~cm}^{-1}$, which could be attributed to the unsymmetrical and symmetrical stretching vibration of $-\mathrm{CH}_{2}$, respectively. These results indicated that the organic macromolecular chains of intercalation agent penetrated into the silicate interlayer areas of the Mg-MMT.

\section{Effect of calcination catalyst}

MMT needs to be dried at a high temperature after modification, so it is necessary to investigate the influence of the calcination temperature on dimerization. Using T-MMT as the representative example, the effects of calcination temperature on the yield and selectivity of the 


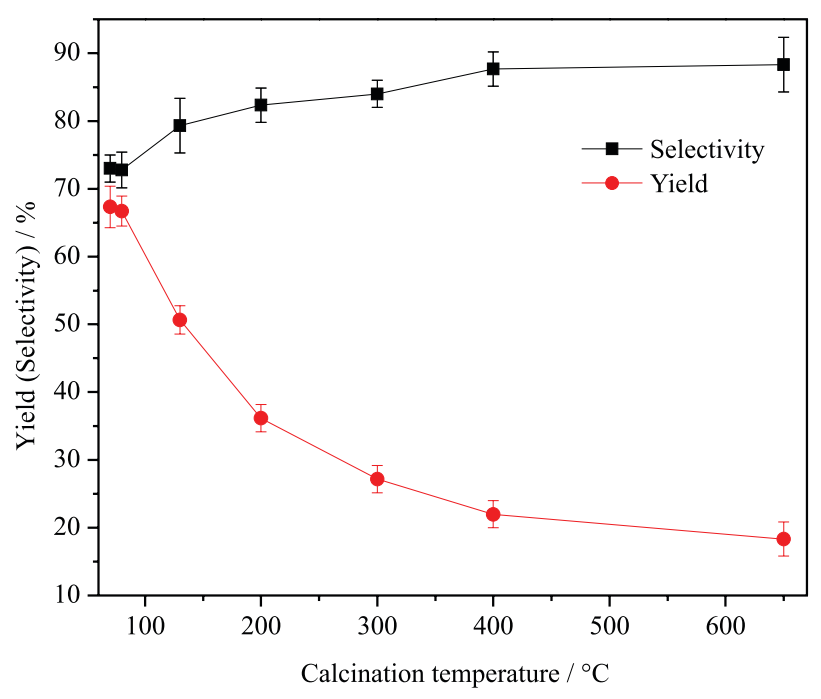

Figure 3. Effect of calcination temperature on the yield and the selectivity.

dimerization reaction were studied, and the results are presented in Figure 3.

It can be seen from Figure 3 that the yield of dimer acid is not affected when the calcination temperature of MMT is lower than $80^{\circ} \mathrm{C}$. As the calcination temperature of MMT increases from 80 to $500{ }^{\circ} \mathrm{C}$, the yield of dimer acid decreases gradually. When the calcination temperature reaches $650{ }^{\circ} \mathrm{C}$, the catalytic performance of T-MMT is basically lost. However, the opposite trend is observed for selectivity of dimer acid; the selectivity increases with the rise in temperature, likely because MMT loses free water at $130{ }^{\circ} \mathrm{C}$, interlayer water at $230{ }^{\circ} \mathrm{C}$, and crystallization water at $637{ }^{\circ} \mathrm{C}$. These assumptions were confirmed by the TGA and DTG (derivative thermogravimetric analysis) results, which are shown in Figure 4.

The DTG curves of Mg-MMT shows two peaks: one peak at $67{ }^{\circ} \mathrm{C}$ was attributed to the physically adsorbed water, another peak at $637^{\circ} \mathrm{C}$ was owing to the structural water. Moreover, the DTG curves of O-MMT and T-MMT show five steps, and the peaks at 57 and $658^{\circ} \mathrm{C}$ were also related to the physically adsorbed water and structural water, respectively, while the three mass-loss steps from 200 to $450^{\circ} \mathrm{C}$ were mainly resulted from the thermal degradation of CTAB. Generally, the combination of CTAB and MMT is due to surface absorption, interlayer-adsorption and intercalation, leading to different degradation temperature of organic MMT. However, in this paper, there was no decomposition of surfactant physically absorbed on the external surface of the organic MMT around 150 to $200^{\circ} \mathrm{C}$. The thermal degradation process at $255^{\circ} \mathrm{C}$ was caused by the surface absorption CTAB molecules, the peak at $337^{\circ} \mathrm{C}$ was caused by the interlayer-adsorption CTAB molecules, and the peak at $421{ }^{\circ} \mathrm{C}$ was attributed to the degradation of intercalated CTAB molecules. Therefore, the result
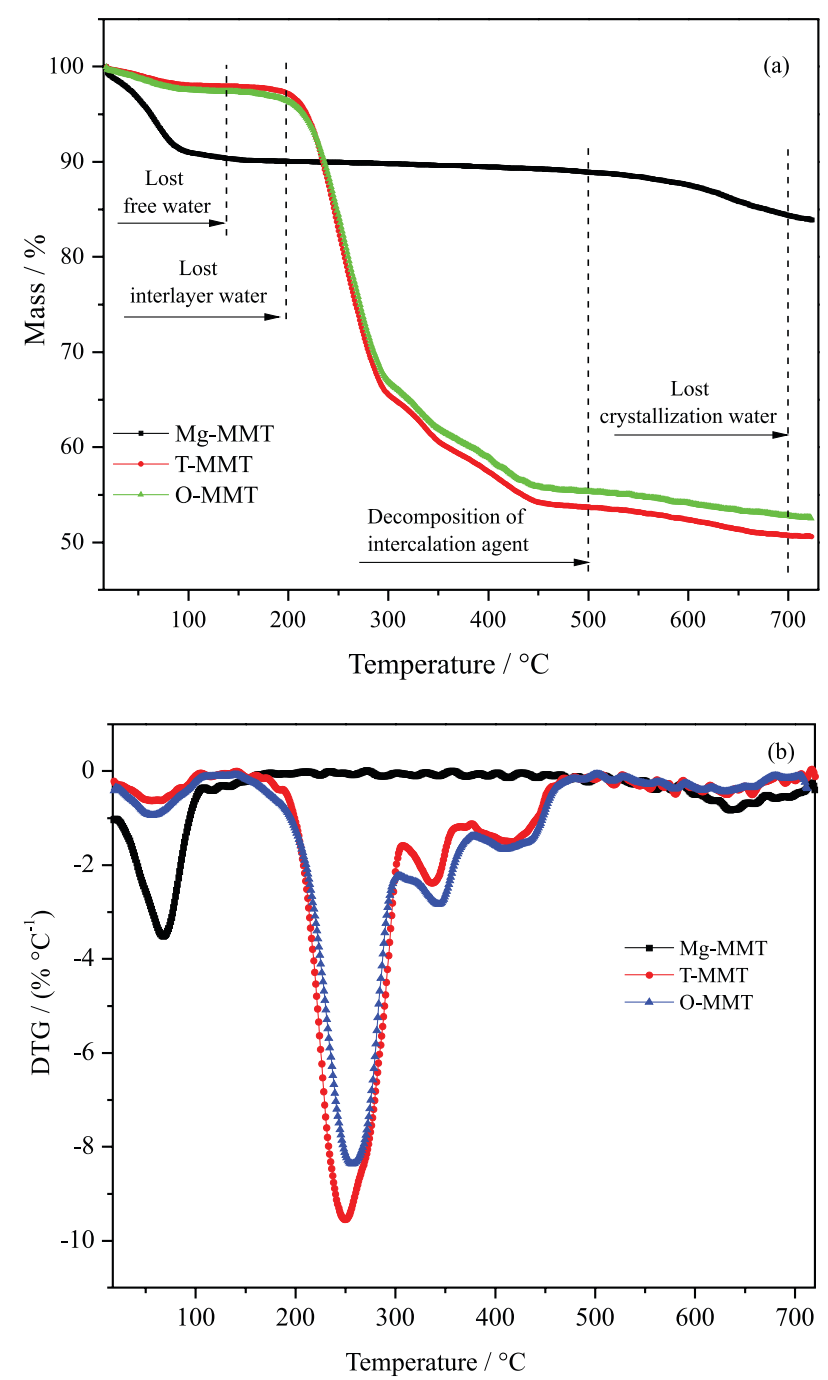

Figure 4. (a) TGA and (b) DTG curves of Mg-MMT, O-MMT and T-MMT.

of TGA-DTG analysis demonstrates that the inorganic cations in montmorillonite were replaced by $\mathrm{CTAB}$, i.e., $\mathrm{CTAB}$ molecules have been successfully inserted into the layers of MMT.

It is generally believed that the interlayer ions of MMT can form a Brønsted-Lewis acid center with one or two layers of water molecules adsorbed by the MMT lattice layer, which can promote the dimerization reaction. Therefore, the moisture content of MMT has a great influence on its catalytic activity. With the increase in calcination temperature, the free water and interlayer water of MMT are continuously removed, thus seriously affecting the catalytic performance of MMT. The lowered catalytic activity of MMT results in fewer side reactions of the reaction system, which causes the increase in selectivity with the increase in temperature. Therefore, the O-MMT and T-MMT cannot be calcined at high temperature to stabilize the crystal structure. 


\section{BET analysis}

$\mathrm{N}_{2}$ adsorption-desorption isotherms are shown in Figure 5 and the specific surface area results can be found in Table 2. As can be seen from Figure 5, $\mathrm{N}_{2}$ adsorption and desorption isotherms are type-IV style, which coincides with the lamellar structure of MMT. The specific surface area of the three kinds of MMT is quite different. The specific surface area of T-MMT is $165.30 \mathrm{~m}^{2} \mathrm{~g}^{-1}$, which is nearly 13 times that of Mg-MMT. The dense aggregation between MMT particles provided a larger specific surface area, and the loose accumulation between MMT particles showed a smaller specific surface area. However, according to the catalytic properties displayed in Table 2, the specific surface area of three kinds of MMT is not positively related to its catalytic activity, so that the specific surface area of MMT is not the main factor affecting its catalytic activity.

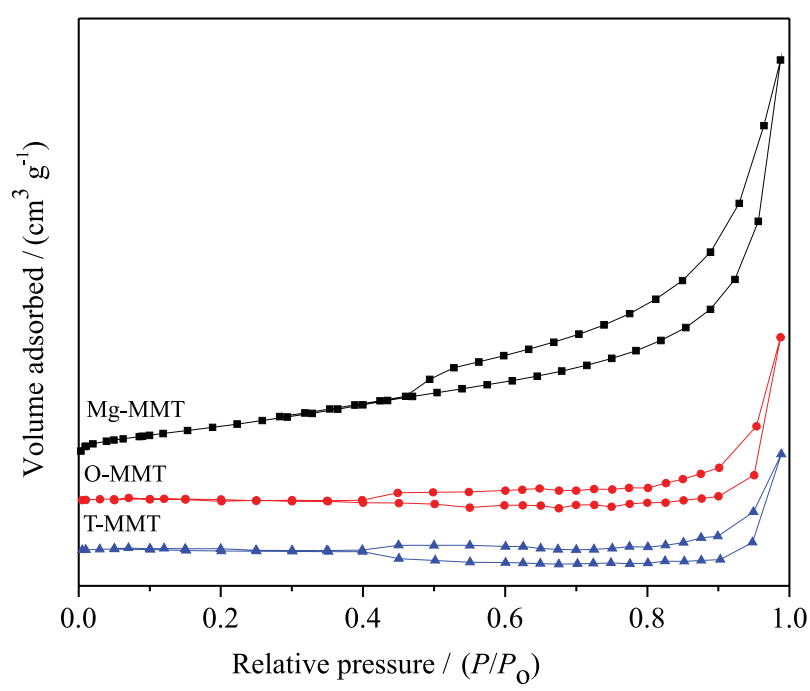

Figure 5. $\mathrm{N}_{2}$ adsorption/desorption isotherm of MMT.

\section{SEM and TEM analysis}

The surface morphologies of Mg-MMT before (Figure 6a) and after (Figures $6 \mathrm{~b}$ and $6 \mathrm{c}$ ) modification by CTAB are depicted in Figure 6. The SEM micrographs show that MMT with further modification resulted in more loosened and disordered organo-clays, attributing to the expansion of basal distance after intercalation reactions.
TEM images of Mg-MMT, O-MMT and T-MMT are shown in Figures 6d, 6e and 6f, respectively. As observed from the figures, O-MMT and T-MMT displayed the typical and well-ordered layer structure like that of Mg-MMT, and the basal distance also expanded further, which was much larger and more obvious than that of $\mathrm{Mg}$-MMT. Thus, the cationic surfactant of CTAB was intercalated successfully into the clay, which gives an indication for the easier adsorption of unsaturated fatty acids.

\section{Effect of dispersibility}

The purpose of MMT modification was to improve the compatibility and dispersibility of MMT in unsaturated fatty acids. In order to investigate the dispersion ability of MMT and optimize the parameters for improving the polymerization of unsaturated fatty acids, deionized water and oleic acid were selected as the dispersion media. The Mg-MMT, O-MMT and T-MMT dispersions were placed into three colorimetric tubes, from left to right. The experimental results are presented in Figure 7.

It can be seen in Figure $7 \mathrm{a}$ that the unmodified Mg-MMT has good hydrophilicity and is not layered for $7 \mathrm{~h}$. It still shows good dispersibility in deionized water up to $24 \mathrm{~h}$. Whereas, the modified O-MMT and T-MMT are essentially stratified at $3 \mathrm{~h}$, and the dispersibility of modified MMT in deionized water is less than that of Mg-MMT. As seen from Figure 7b, Mg-MMT was fully precipitated from oleic acid in $7 \mathrm{~h}$; O-MMT showed complete precipitation in $24 \mathrm{~h}$, but was still slightly dispersed in oleic acid; and T-MMT showed complete precipitation in $48 \mathrm{~h}$. Thus, the dispersibility of the samples in oleic acid follows the order: T-MMT $>$ O-MMT $>$ Mg-MMT. This trend is consistent with the results of the catalytic performance of the three samples, as discussed in previous section. As the Mg-MMT is modified, it is transformed from being hydrophilic to hydrophobic in the O-MMT and T-MMT samples, which accounts for the improved dispersibility.

\section{Effect of the surface acidity}

The Brønsted and Lewis acids of the three catalysts were

Table 2. The specific surface area and catalytic properties of MMT

\begin{tabular}{lcccc}
\hline MMT & Specific surface area $/\left(\mathrm{m}^{2} \mathrm{~g}^{-1}\right)$ & Specific pore volume $/\left(\mathrm{cm}^{3} \mathrm{~g}^{-1}\right)$ & Selectivity of dimer acids $/ \%$ & Yield of dimer acids $/ \%$ \\
\hline Mg-MMT & 12.84 & 0.012 & 73.10 & 32.69 \\
O-MMT & 165.30 & 0.20 & 72.19 & 50.86 \\
T-MMT & 109.78 & 0.05 & 72.77 & 68.03 \\
\hline
\end{tabular}

O-MMT: one-step intercalated MMT; T-MMT: two-step intercalated MMT. 


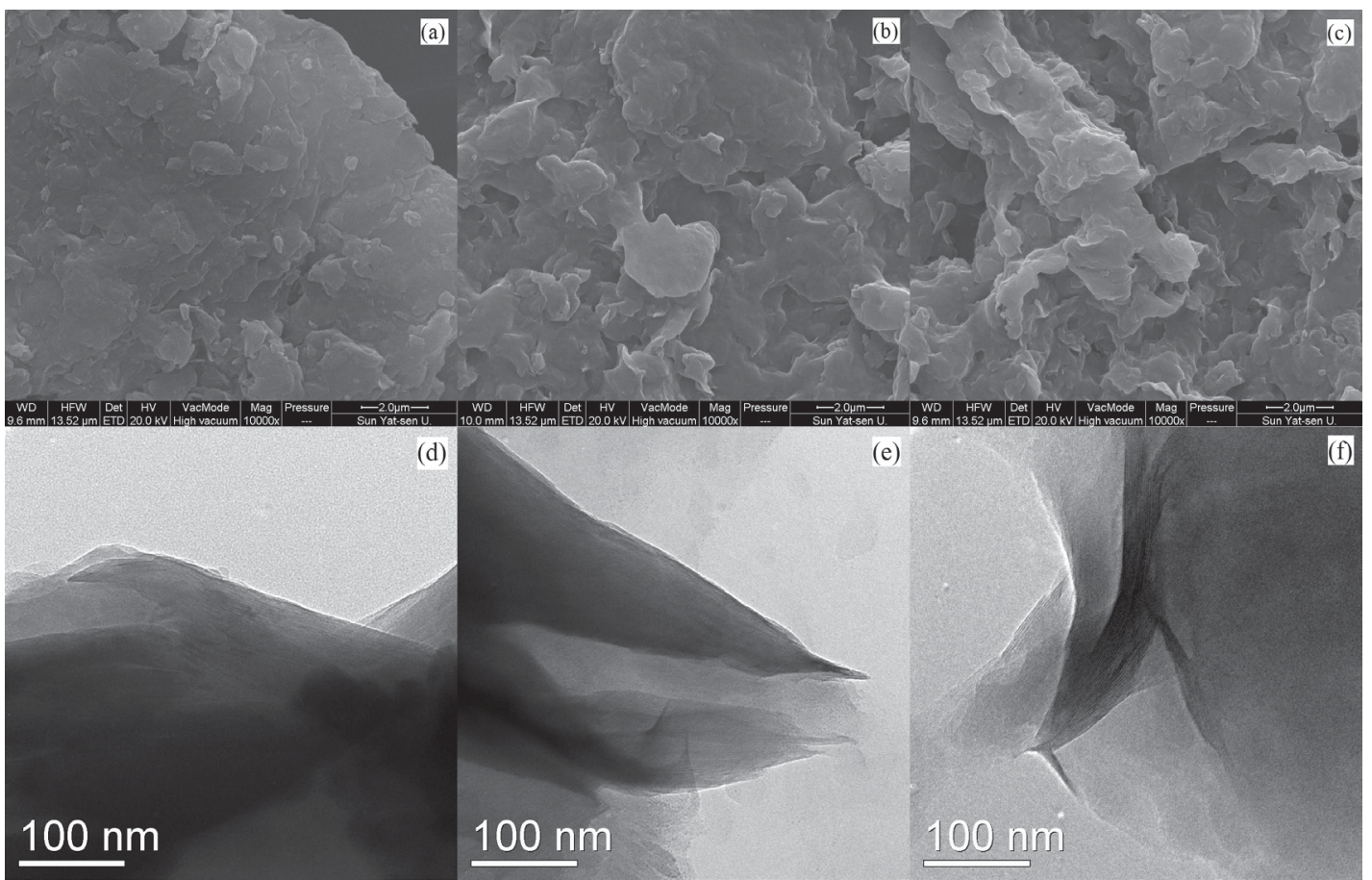

Figure 6. (a, b, c) SEM and (d, e, f) TEM analysis of (a, d) Mg-MMT; (b, e) O-MMT and (c, f) T-MMT.

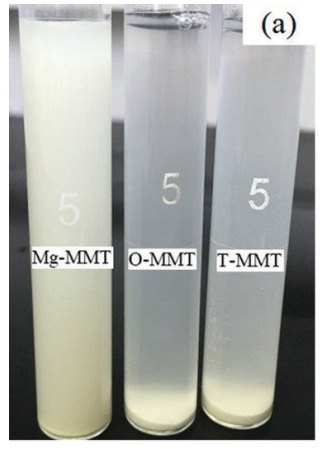

$3 \mathrm{~h}$

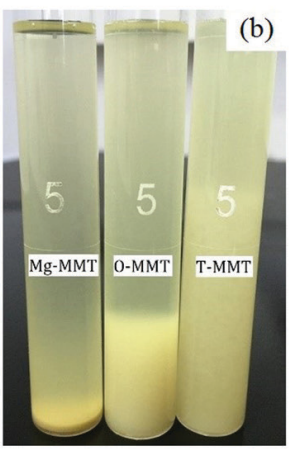

$3 \mathrm{~h}$

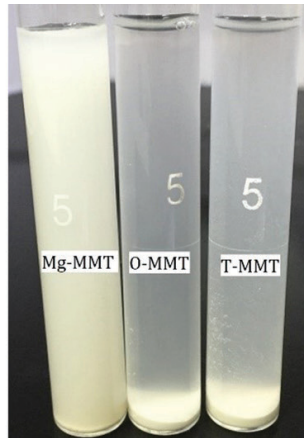

$7 \mathrm{~h}$

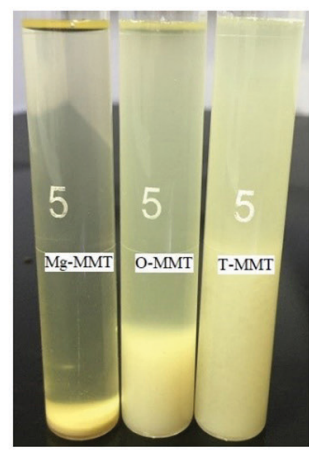

$7 \mathrm{~h}$

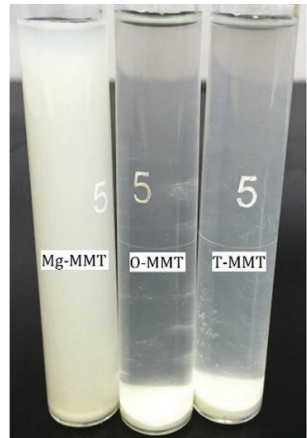

$24 \mathrm{~h}$

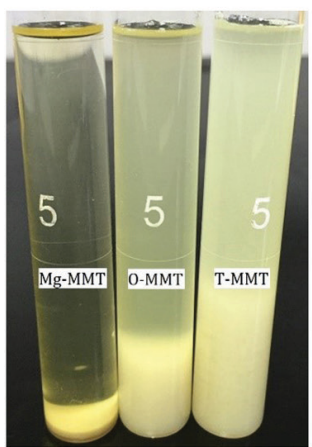

$24 \mathrm{~h}$

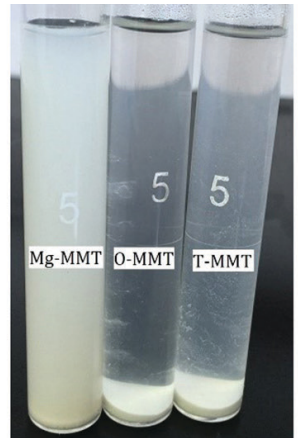

$48 \mathrm{~h}$

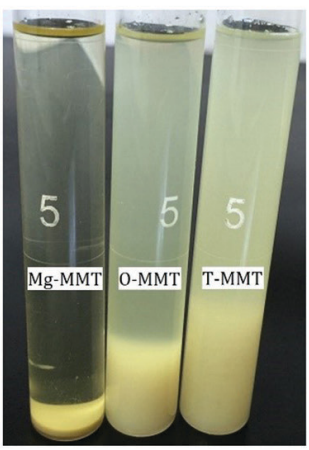

$48 \mathrm{~h}$

Figure 7. Dispersibility of Mg-MMT and modified MMT in (a) deionized water and (b) oleic acid.

characterized by pyridine adsorption infrared spectroscopy. The infrared spectra are shown in Figure 8.

The band at $1605 \mathrm{~cm}^{-1}$ is the characteristic absorption peak of pyridine. The band at $1448 \mathrm{~cm}^{-1}$ is the characteristic absorption peak of pyridine forming coordination bond with the center of Lewis acid. However, the characteristic absorption band at $1545 \mathrm{~cm}^{-1}$ of pyridine ion formed by pyridine and Brønsted acid is not obvious in the spectrum. The band at $1487 \mathrm{~cm}^{-1}$ is a superposition of the absorption peaks formed by the Brønsted and the Lewis acids 


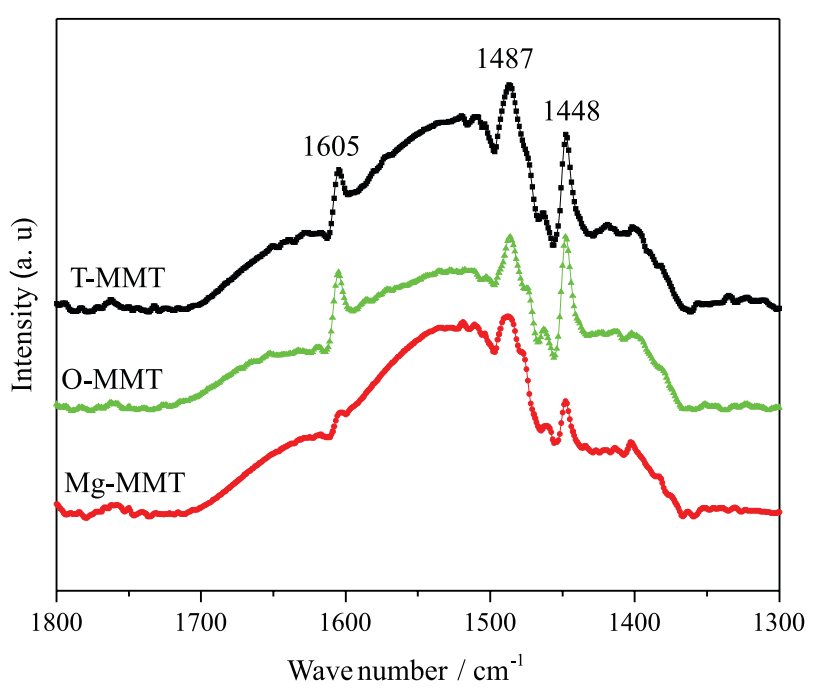

Figure 8. The pyridine adsorption infrared spectra of three kinds of MMT.

respectively with pyridine. The molar ratio of the Lewis acid center $\left(\mathrm{x}_{\mathrm{L}}\right)$ to the $\mathrm{Br} ø$ nsted acid center $\left(\mathrm{x}_{\mathrm{B}}\right)$ is calculated according to the Anderson formula (equation 3), and the results are shown in Table 3.

$\frac{\mathrm{x}_{\mathrm{L}}}{\mathrm{x}_{\mathrm{B}}}=\frac{\left(\mathrm{E}_{1487}^{\mathrm{B}} / \mathrm{E}_{1487}^{\mathrm{L}}\right)\left(\mathrm{E}_{1487}^{\mathrm{L}} / \mathrm{E}_{1448}\right) \mathrm{A}_{1448}}{\mathrm{~A}_{1487}-\left(\mathrm{E}_{1487}^{\mathrm{L}} / \mathrm{E}_{1448}\right) \mathrm{A}_{1448}}$

where $\mathrm{A}$ is the absorbance and $\mathrm{E}$ is the extinction coefficient.

According to Basila and Kantner, ${ }^{22} \mathrm{E}_{1487}^{\mathrm{L}} / \mathrm{E}_{1448}$ is 0.25 , and $\mathrm{E}_{1487}^{\mathrm{B}} / \mathrm{E}_{1487}^{\mathrm{L}}$ is 5.8, which was used to calculate the ratio of $x_{L} / x_{B}$. Based on the ratio of $x_{L} / x_{B}$ and the catalytic performance, it could be concluded that the catalytic activity of T-MMT with high Lewis acid concentration was significantly higher and the yield of dimer acid was also higher. This is explained by the fact that the Lewis acid center plays the key role in the polymerization of unsaturated fatty acids. During the process of dimerization, the Lewis acid center can promote the cracking and the transfer of hydrogen in the oleic acid molecule, resulting in conjugated linoleic acid and carbenium ions, as shown in Figure 9.

In the dimerization process of linoleic acid, the Lewis acid center can accelerate the formation of conjugated linoleic acid by isomerization of the unconjugated linoleic acid double bond, as shown in Figure 10. These two steps are considered to be the rate limiting steps in the mechanism of oleic acid and linoleic acid polymerization, respectively. Thus, T-MMT with more Lewis acid sites shows better catalytic performance in the dimerization of unsaturated fatty acids.

\section{Effect of $x_{A} / x_{S i}$ on the surface of catalyst}

SEM-EDS was used to identify the surface morphology and elemental composition of MMT, as shown in Figure 11 and Table 4. Figures 11a, 11b and 11c show the SEM micrographs and elemental analysis results of single particles of Mg-MMT, O-MMT and T-MMT, respectively. Mg-MMT is a compact spherical particle. After modification, the O-MMT particles became disordered. The compact spheres expanded and multilayer

Table 3. Surface acidities and catalytic properties of montmorillonite (MMT) samples

\begin{tabular}{lcccccc}
\hline MMT & $\mathrm{x}_{\mathrm{L}} /\left(\mathrm{mmol} \mathrm{g}^{-1}\right)$ & $\mathrm{x}_{\mathrm{L}} / \mathrm{x}_{\mathrm{B}}$ & $\mathrm{x}_{\mathrm{Al}} / \mathrm{x}_{\mathrm{Si}}$ & $\mathrm{x}_{\mathrm{Mg}}$ & Selectivity of dimer acids / \% & Yield of dimer acids / \% \\
\hline Mg-MMT & 0.011 & 0.5273 & 0.5010 & 0.22 & 73.10 & 32.69 \\
O-MMT & 0.087 & 1.2609 & 0.4306 & 0.12 & 72.19 & 50.86 \\
T-MMT & 0.124 & 2.1368 & 0.4434 & 0.08 & 72.77 & 68.03 \\
\hline
\end{tabular}

x: molar content; O-MMT: one-step intercalated MMT; T-MMT: two-step intercalated MMT.

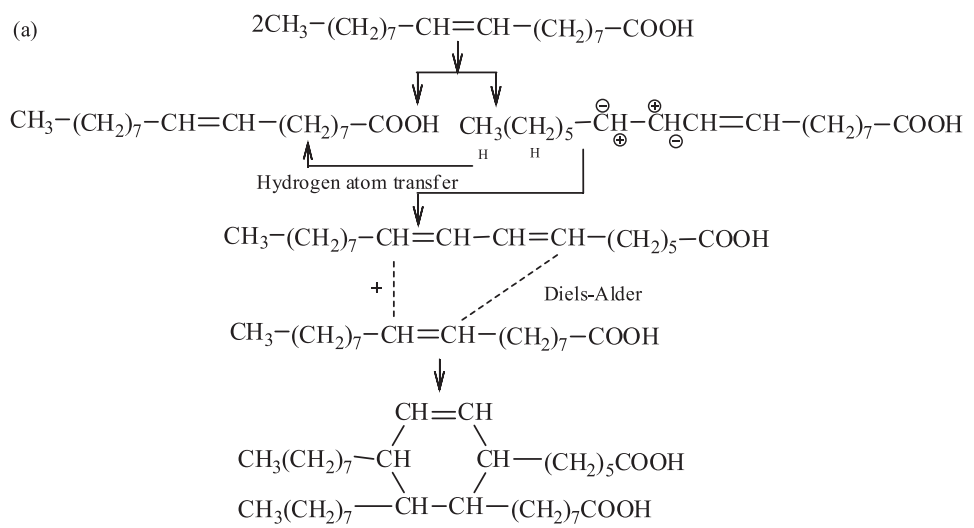

(b) $\mathrm{CH}_{3}-\left(\mathrm{CH}_{2}\right)_{7}-\mathrm{CH}=\mathrm{CH}-\left(\mathrm{CH}_{2}\right)_{7}-\mathrm{COOH}$

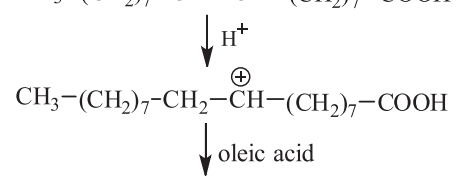

$\mathrm{CH}_{3}-\left(\mathrm{CH}_{2}\right)_{7}-\mathrm{CH}_{2}-\mathrm{CH}-\left(\mathrm{CH}_{2}\right)_{7}-\mathrm{COOH}$

$\mathrm{CH}_{3}-\left(\mathrm{CH}_{2}\right)_{7}-\mathrm{CH}-\mathrm{CH}-\left(\mathrm{CH}_{2}\right)_{7}-\mathrm{COOH}$ $\downarrow-\mathrm{H}^{+}$

$\mathrm{CH}_{3}-\left(\mathrm{CH}_{2}\right)_{7}-\mathrm{CH}_{2}-\mathrm{CH}-\left(\mathrm{CH}_{2}\right)_{7}-\mathrm{COOH}$

$\mathrm{CH}_{3}-\left(\mathrm{CH}_{2}\right)_{7}-\mathrm{CH}=\stackrel{\mathrm{C}}{\mathrm{C}}-\left(\mathrm{CH}_{2}\right)_{7}-\mathrm{COOH}$

Figure 9. The polymerization mechanism of oleic acid under the catalytic conditions of MMT. (a) Produce conjugated linoleic acid; (b) produce carbonium ion of olefins. 


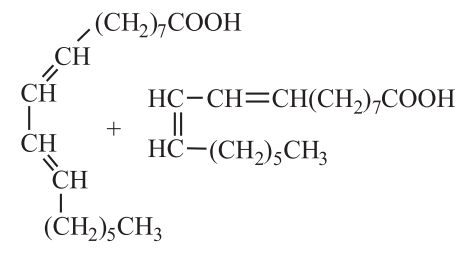

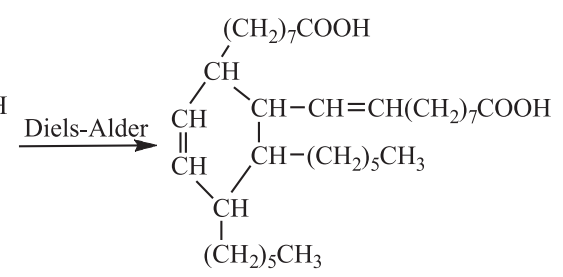

Figure 10. The polymerization mechanism of linoleic acid under the catalytic conditions of MMT.

structures could be observed on the surfaces of the particles. The structural evolution was primarily due to the change in the basal distance of MMT. Compared to O-MMT, the multilayer structure of T-MMT is more loose, because the layer spacing of MMT is further expanded after the second modification. These SEM results are in agreement with XRD results.

The molar contents of $\mathrm{Al}$ and $\mathrm{Si}$ elements on different MMT surfaces were determined by EDS, and the ratio of the contents is given in Tables 3 and 4. The results show that the difference between the molar $\mathrm{x}_{\mathrm{Al}} / \mathrm{x}_{\mathrm{Si}}$ ratios of the three kinds of MMT is very small, and all the $\mathrm{x}_{\mathrm{Al}} / \mathrm{x}_{\mathrm{Si}}$ ratios lie within the range of $0.4306-0.5010$. It is considered that the $\mathrm{x}_{\mathrm{Al}} / \mathrm{x}_{\mathrm{Si}}$ difference of the three kinds of MMT is not due to Al content.

The molar content of $\mathrm{Mg}$ in Table 3 decreases with the increase in number of intercalation steps, indicating that $\mathrm{Mg}^{2+}$ is replaced by CTAB and the interlayer distance is enlarged, which is also consistent with the XRD results.
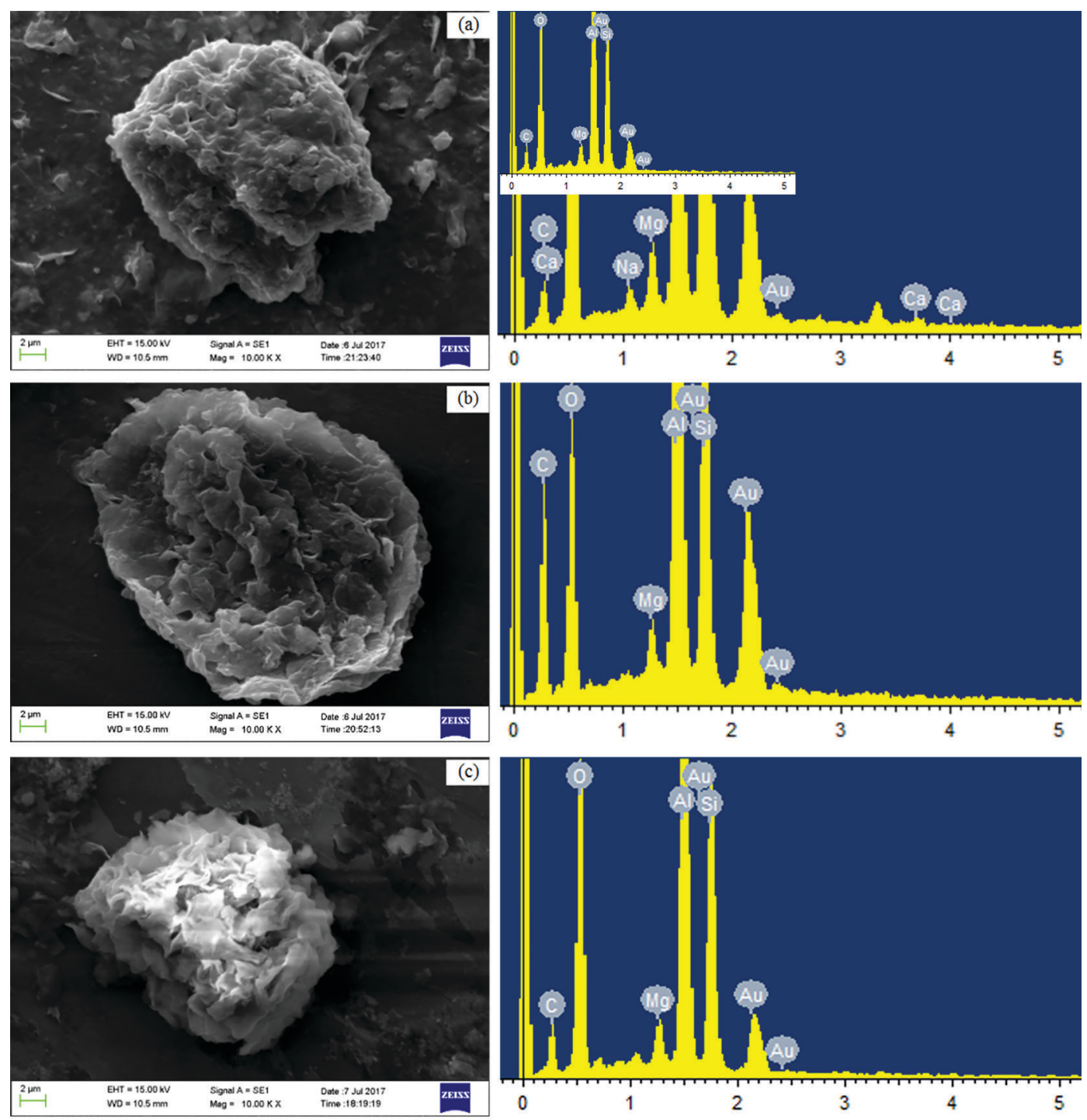

Figure 11. SEM-EDS images of (a) Mg-MMT; (b) O-MMT and (c) T-MMT. 
Table 4. Element content of montmorillonite (MMT) samples

\begin{tabular}{lccc}
\hline $\begin{array}{l}\text { Element } \\
\text { content / } \%\end{array}$ & Mg-MMT & O-MMT & T-MMT \\
\cline { 2 - 4 } $\mathrm{C}$ & 15.29 & 29.45 & 27.99 \\
$\mathrm{O}$ & 52.16 & 41.24 & 42.85 \\
$\mathrm{Na}$ & 0.53 & 0 & 0 \\
$\mathrm{Mg}$ & 1.45 & 1.08 & 0.71 \\
$\mathrm{Al}$ & 9.76 & 8.18 & 8.41 \\
$\mathrm{Si}$ & 18.79 & 18.31 & 18.28 \\
$\mathrm{Ca}$ & 0.19 & 0 & 0 \\
$\mathrm{Au}$ & 1.85 & 1.75 & 1.76 \\
\hline Total & 100.02 & 100.01 & 100.00 \\
\hline
\end{tabular}

$\mathrm{Au}$ is sprayed on the sample for testing.

\section{Reusability of catalysts}

The reusability of the catalyst is shown in Figure 12. It can be seen that after repeatedly used for five times the yield decreases slightly from 68.03 to $57.51 \%$ for the T-MMT, and 50.86 to $33.87 \%$ for the O-MMT. Therefore, T-MMT catalysts are the best choice for the repeated usability. However, the yield of Mg-MMT decreased from 32.37 to $9.09 \%$, suggesting a poor performance of its reusability.

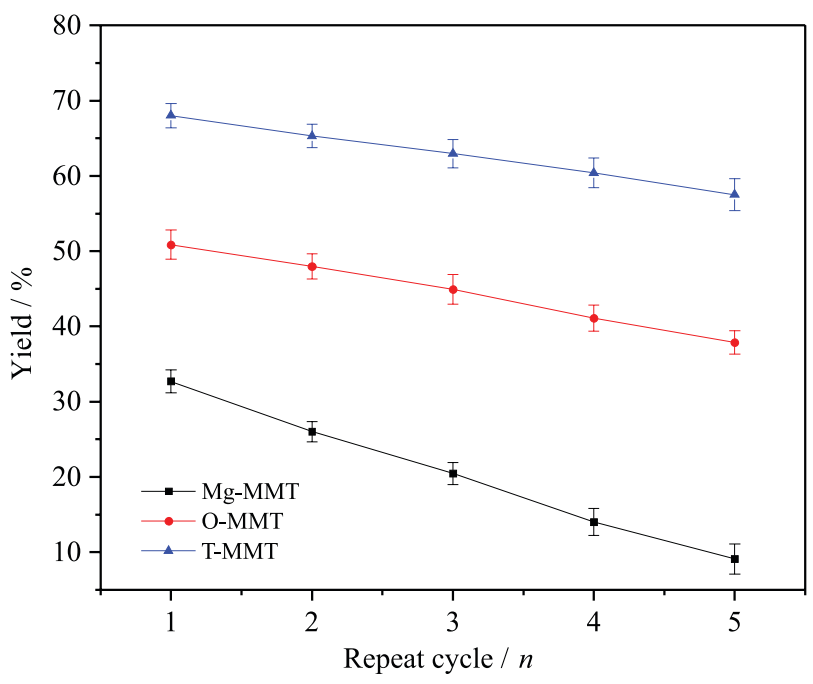

Figure 12. Reusability of MMTs.

\section{Conclusions}

The effects of structure and composition of MMT catalyst on the dimerization of unsaturated fatty acids were examined by characterizing the specific surface area, texture and morphology, surface acidity and dispersibility of different MMTs. The results show that the specific surface area has little effect on the catalytic activity of MMT. The interlayer spacing of the MMT crystals is favorable for the formation and diffusion of dimerization products, which helps to improve the yield of dimer acids. The higher Lewis acid density on the surface of MMT is favorable for the hydrocracking and hydrogen transfer processes of oleic acid, which in turn accelerates the isomerization of unconjugated linoleic acid to form conjugated linoleic acid. Therefore, higher $\mathrm{x}_{\mathrm{L}} / \mathrm{x}_{\mathrm{B}}$ ratios can give higher yields of dimer acid. After modification of MMT, its lipophilicity is improved, and its dispersibility is better in the fatty acid. The improved dispersibility is favorable for the unsaturated fatty acid molecules to enter the MMT interlayer, thus improving the yield of dimer acids. The molar ratio of $\mathrm{x}_{\mathrm{Al}} / \mathrm{x}_{\mathrm{Si}}$ in MMT remains almost unchanged, before and after modification, and the $\mathrm{Al}$ element is not the source of the Lewis acid. Thus, the $\mathrm{x}_{\mathrm{Al}} / \mathrm{x}_{\mathrm{Si}}$ molar ratio is not a factor affecting the dimerization reaction.

\section{Acknowledgments}

The work is supported by the Natural Science Foundation of China (No. 31401526). We are grateful to the anonymous reviewers for their valuable comments and suggestions which helped to improve the quality of the paper. Thanks for the reviewers' and editor's attention to our manuscript and we would wish to appreciate their detailed and professional advices again.

\section{References}

1. Brütting, R.; Spiteller, G.; Eur. J. Lipid Sci. Technol. 2010, 95, 193.

2. Reulier, M.; Matadi, R. B.; Walsh, Z. K.; Vaudemont, R.; Avérous, L.; J. Appl. Physiol. 2016, 134, 44610.

3. Burg, D. A.; Kleiman, R.; J. Am. Oil Chem. Soc. 1991, 68, 600.

4. Yu, F.; Saha, P.; Suh, P. W.; Kim, J. K.; J. Appl. Polym. Sci. 2015, 132, 681.

5. Gogoi, G.; Gogoi, S.; Karak, N.; Prog. Org. Coat. 2017, 112, 57.

6. Keersmaecker, M. D.; Berg, O. V. D.; Verbeken, K.; Depla, D.; Adriaens, A.; J. Electrochem. Soc. 2015, 162, C167.

7. Pavlin, M. S.; US pat. 80583862011 (CA 273:6882A1).

8. Freitas, R. F. R.; Klein, C.; Pereira, M. P.; Duczinski, R. B.; Einloft, S.; Seferin, M.; Ligabue, R.; J. Adhes. Sci. Technol. 2015, 29, 1860.

9. Tolvanen, P.; Mäkiarvela, P.; Kumar, N.; Eränen, K.; Sjöholm, R.; Hemming, J.; Holmbom, B.; Salmi, T.; Murzin, D. Y.; Appl. Catal. A 2007, 330, 1.

10. Mäkiarvela, P.; Mikkola, M.; Hemming, J.; Eränen, K.; Willför, S.; Murzin, D. Y.; J. Am. Oil Chem. Soc. 2014, 91, 1035.

11. Zhang, S.; Zhou, J.; Zhang, Z. C.; Catal. Lett. 2009, 127, 33. 
12. Tolvanen, P.; Mäkiarvela, P.; Eränen, K.; Wärnå, J.; Holmbom, B.; Salmi, T.; Murzin, D. Y.; J. Am. Oil Chem. Soc. 2008, 85, 567.

13. Reaume, S. J.; Ellis, N.; J. Am. Oil Chem. Soc. 2011, 88, 661.

14. Koster, R. M.; Bogert, M.; Leeuw, B. D.; Poels, E. K.; Bliek, A.; J. Mol. Catal. A: Chem. 1998, 134, 159.

15. Liu, S. W.; Zhou, H. X.; Yu, S. T.; Song, Z. Q.; Chem. Eng. J. 2011, 174, 396.

16. Utracki, L. A.; Sepehr, M.; Boccaleri, E.; Polym. Adv. Technol. 2010, 18,1 .

17. Č́̌čel, B.; Komadel, P.; Nigrin, M.; Collect. Czech. Chem. Commun. 1992, 57, 1666.
18. Escudero, J.; Notario, B.; Jimenez, C.; Rodriguez-Perez, M. A.; J. Appl. Polym. Sci. 2016, 133, 43432.

19. Su, X. L.; Ma, L. Y.; Wei, J. M.; Zhu, R. L.; Appl. Clay Sci. 2016, 132-133, 261.

20. Sánchez-Martín, M. J.; Dorado, M. C.; del Hoyo, C.; RodríguezCruz, M. S.; J. Hazard. Mater. 2008, 150, 115.

21. Hussin, F.; Aroua, M. K.; Wan, M. A. W. D.; Chem. Eng. J. 2011, 170, 90.

22. Basila, M. R.; Kantner, T. R.; J. Phys. Chem. 1967, 70, 19.

Submitted: September 3, 2017

Published online: February 16, 2018 